\title{
Desastres, memorias y bienestar de lugar en Dichato, Chile
}

\author{
Disasters, memories and well-being of the place in Dichato, Chile
}

\author{
Pablo Alcota ${ }^{1}$ y Andrea Aravena-Reyes ${ }^{2}$ \\ ${ }^{1}$ Psicólogo. Doctor en Psicología, Universidad de Concepción. Concepción, Chile. Profesor en la \\ Escuela de Psicología, Facultad de Educación y Humanidades, Universidad del Bío-Bío, Chile. \\ E-mail: p.alcota@gmail.com
}

${ }^{2}$ Antropóloga. Doctora en Antropología Social y Etnología. Profesora de grado y postgrado en la Facultad de Ciencias Sociales, Universidad de Concepción, Chile.

La investigación que se informa fue financiada por la Comisión Nacional de Investigación Científica y Tecnológica, Chile. CONICYT/Doctorado Nacional/21160131.

Facultad de Ciencias Sociales, Universidad de Concepción.

Concepción, Chile.

\section{Resumen}

El trabajo que se presenta entrega aportes para comprender el impacto de desastres naturales o antrópicos, como terremotos, maremotos, huracanes, terrorismo, entre otros, producidos por efectos de la acción de fenómenos naturales o acciones humanas, en personas. Su objetivo es comprender el impacto del terremoto $8.8^{\circ}$ y tsunami del 27 de febrero de 2010, en Dichato, localidad costera al sur de Chile, a partir de la necesidad de generar conocimiento sobre los alcances traumáticos de lo consignado en el bienestar en ese lugar. Se trabajó a partir de los relatos de habitantes sobrevivientes sobre su trayectoria de vida en el lugar, en quienes emerge el recuerdo y superposición de la experiencia traumática de la vivencia del terremoto $\mathrm{y}$ tsunami mencionados, a lo que fue la dictadura militar de Augusto Pinochet de 19731990, como acontecimiento desastroso equivalente en sus vidas. Los resultados amplían la comprensión de desastre, más allá de sus alcances materiales, individuales o de corto plazo. Concluimos que el impacto socio-histórico-emocional, el daño al sujeto social y su bienestar de lugar, son aspectos que deben considerarse ante las transformaciones $y$ cambios en el lugar de vida, conociendo y comprendiendo las respuestas colectivas que puedan emerger.

Palabras clave: Desastres; Memoria colectiva; Bienestar; Bienestar de lugar; Chile.

\begin{abstract}
In this article, we provide contributions to understand the impact of natural or anthropic disasters, like earthquakes, tsunamis, terrorism, and so on, caused by the effects of natural disasters or human actions over people. Our objective is to understand the impact of the $8.8^{\circ}$ earthquake and tsunami of February $27^{\text {th }}$, 2010 , in Dichato, in the south coast of Chile. The interest is to generate knowledge about the effects of traumatic events in the well-being of the people who live in that place. The social and health sciences need to integrate other methods inn their research, in order to study the impact of traumatic events on the well-being of people. We worked with surviving inhabitants that told us about their lives in that place and their life stories. In these accounts, there was
\end{abstract}


some remembrances and emotional superpositions between the traumatic experiences of the earthquake and the tsunami, and the military dictatorship of Augusto Pinochet from 19731990 in Chile, considered as a similar disastrous event in their lives. The results expand the understanding about disasters, beyond its material, individual or short-term scope. We conclude that the socio-historical emotional impact, social suffering and well-being of the place are aspects that should be considered in the face of transformations and changes in the place of living, knowing and understanding the collective responses that can emerge.

Keywords: disasters; collective memory; wellbeing; well-being of the place; Chile.

\section{Introducción}

La integración de herramientas comprensivas sobre el vínculo con lugares significativos y los procesos de memoria colectiva (Aravena, 2003; Baeza, 2011; Cárdenas, Páez, Rimé, Bilbao y Asún, 2014; Halbwachs, 2004; Low y Altman, 1992; Muller y Bermejo, 2016; Piper-Shafir, Fernández-Droguett e Íñiguez-Rueda, 2013; Sepúlveda-Galeas, Sepúlveda, Piper y Troncoso, 2015) ayuda a comprender el impacto de desastres naturales o antrópicos (Cardona, 1993; Jha, 2010; Romero y Maskrey, 1993), que pueden llegar a promover o acentuar graves transformaciones sociales, físicas e incluso respuestas ante tales transformaciones. Es de gran importancia comprender las implicaciones de drásticos acontecimientos, como los desastres, que cambian los lugares que valoramos, en torno a los cuales se construyen lazos que aportan a nuestra experiencia de salud y bienestar (Duff, 2009; Quinn y Halfcare, 2014).

El estudio del impacto de estos acontecimientos en el sujeto social, debe considerar esferas psicosociales que se ven gravemente afectadas ante la ocurrencia de cambios en el lugar de vida, que algunos autores han denominado la dimensión humana de los desastres (Sapiains y Ugarte, 2017a, 2017b).
Se entiende por sujeto social al sujeto en sociedad, es decir, al sujeto en relación intergrupal, conformándose un colectivo que produce conocimiento social sobre ciertos acontecimientos, construyendo lecturas que le posibilitan sentir, actuar y comprender la realidad social. Es por ello que profundizar la comprensión del impacto del terremoto del 27 de febrero de 2010 (27F), significó arribar a una dimensión de análisis que se denominó bienestar de lugar y que considera el bienestar desde una mirada profunda, situada y significada, donde el sujeto social y su bienestar son especialmente relevantes.

La literatura científica respecto al impacto de desastres, desde una perspectiva individual, plantea la necesidad de poner atención en grupos específicos, como lo son miembros de minorías étnicas y grupos de bajo estatus socioeconómico, ya que muestran un aumento en los efectos adversos en su salud mental, en comparación con la población general (Norris, 2006; Norris, Friedman, Watson, Byrne, Diaz y Kaniasty, 2002). Igualmente, como afectados directos e indirectos de un desastre o catástrofe, se ha encontrado un aumento de ideas suicidas y comportamiento autodestructivo (Kõlves, Kõlves y De Leo, 2013; Panagioti, Gooding y Tarrier, 2009).

Chile forma parte de una zona geográfica de alta vulnerabilidad asociada al cambio climático (Oficina de Cambio Climático, 2014), donde han ocurrido desastres naturales y antrópicos, como terremotos, inundaciones, así como terrorismo de Estado (Carnevali, 2015; Jarvis y Lister, 2014), razones por las cuales requieren profundos esfuerzos comprensivos sobre el impacto de los mismos en la sociedad.

En este sentido, es preocupante que sumado a los desastres consignados, se produzca la paradoja del bienestar en Chile, que da cuenta de elevados índices de bienestar individual, junto a un desfavorable bienestar social y una valoración negativa del Estado, entre otros (Programa de las Naciones Unidas para el Desarrollo, 2012). 
Parte de la problemática en la que se basó la presente investigación se fundamenta en que una serie de drásticos acontecimientos, asociados por ejemplo a acontecimientos naturales que han transformado el lugar de vida, son estudiados a partir de su efecto psicopatológico en las personas afectadas (Cova y Rincón, 2010), comúnmente buscando reconocer el efecto inmediato o de corto plazo de lo aludido, traduciéndose en diagnósticos psicopatológicos parcelados, sin historia, contextualización, ni sujeto social, lo que no permite comprender el impacto de los desastres en el sujeto social. Por ello se estima necesario construir una mirada que integre procesos de memoria colectiva (Baeza, 2011; Halbwachs, 2004; Muller y Bermejo, 2016) en torno a los desastres, pudiendo ayudar a comprender cómo se ha dado sentido a la experiencia y estabilidad a la realidad social, ante acontecimientos altamente desestabilizadores de un orden, que marcaron un pasado, un presente y un devenir de un colectivo (Lykes, 2013; Martín-Baró, 1988).

En la presente investigación el objetivo es comprender el impacto del terremoto $8.8^{\circ}$ y tsunami del 27 de febrero de 2010 , en Dichato, desde la perspectiva de sobrevivientes y habitantes del lugar, buscando nutrir en profundidad y complejidad, la comprensión del impacto de desastres, tanto previo a su ocurrencia, durante y posterior a ellos, y con especial consideración a partir de la necesidad de generar conocimiento que profundice sobre los alcances traumáticos del $27 \mathrm{~F}$ en el bienestar, en ese lugar, lo que convocó a una labor investigativa posicionada (Sandoval, 2013).

Para cumplir con el objetivo propuesto, que se enmarca en una línea de investigación sobre el bienestar, se realizó una aproximación a la problemática desde un diseño de investigación naturalista y de corte cualitativo, reconociendo la dimensión histórica, relacional y discursiva en su construcción, atendiendo a la perspectiva de los participantes.

\section{Antecedentes comprensivos sobre los desastres}

Los desastres, sean antrópicos o naturales (Cardona, 1993; Jha, 2010; Romero y Maskrey, 1993), son graves acontecimientos, producto de la acción u omisión humana, o efectos de la naturaleza, que implican una significativa tensión para las comunidades, o en casos más extremos, la pérdida de vidas, viviendas, trabajos, relaciones sociales, tradiciones ancestrales o la amenaza de desaparición de una cultura o población (Huber y Arnberger, 2015). En otros casos puede significar la relocalización voluntaria o involuntaria (Brown y Perkins, 1992) o facilitar la emergencia de respuestas psicosociales de gran envergadura (Grandón, Acuña, Briese, Chovar, Hernández y Orellanas, 2014).

Estos acontecimientos que aproximan a una población en una situación extrema como la señalada, pueden ser la base del trauma colectivo (Kirmayer, Kienzler, Afana y Pedersen, 2010; Somasundaram, 2007), trauma psicosocial (Lykes, 2013; Martín-Baró, 1988) o sufrimiento social (Pedersen, 2002; Pedersen, Kienzler y Gamarra, 2010), cambiando la vida en el lugar, sin que necesariamente cambie el espacio físico del que se trate.

Los desastres, antrópicos o naturales (Cardona, 1993; Jha, 2010; Romero y Maskrey, 1993), más allá de sus evidentes diferencias, son acontecimientos similares que engloban una serie de distintos acontecimientos que se suceden y resignifican a partir de una imposición de fuerza que violenta y oprime a sujetos o colectivos. En definitiva, un acontecimiento es:

"un punto de inflexión radical, que es, en su auténtica dimensión, invisible. (...) En un acontecimiento, no sólo las cosas cambian: lo que cambia es el propio parámetro por el que medimos los hechos de cambio, es decir, el punto de inflexión cambia el campo entero dentro del cual aparecen los hechos" (Zizek, 2016, p. 155). 


\section{Lugares y confluencia de memorias}

El estudio de las relaciones entre personas y lugares ha sido abordado desde diversos marcos disciplinarios, teorías y métodos (Lewicka, 2011) y específicamente las trayectorias de vida en el lugar (Bailey, Devine-Wright y Batel, 2016; Batel y Devine-Wright, 2015) ayudan a comprender las relaciones que construimos con lugares específicos y respuestas colectivas ante los cambios, como los asociados a un desastre.

En efecto, un lugar constituye un "conjunto de redes personales y características ambientales que juntos trabajan para mejorar o dificultar la experiencia de salud y bienestar" (Duff, 2009, p. 204), donde el vínculo de las personas y grupos hacia lugares trasciende la experiencia interna e individual (Low y Altman, 1992).

Los recuerdos sobre ciertos acontecimientos se vinculan a un proceso de base llamado memoria de lugar (Marcus, 1992) y afloran respecto a la trayectoria, individual y/o grupal, en un determinado lugar, donde confluyen memorias, emociones y lugares. Por otra parte, el objeto de la memoria de lugar, los lugares de memoria (Nora, 1997) convocan a una relación simbólica del sujeto/ grupo con el lugar a través de la memoria (Low, 1992).

En la revisión histórica sobre la trayectoria de vida en un lugar, existe un proceso mnémico que integra un aspecto asociado a la acción de rememorar, y con ello la emergencia de recuerdos emotivos asociados a un lugar, o memoria de lugar, y otro asociado al lugar de memoria, que es el objeto o lugar específico que se rememora, y que estimula dicha acción de recordar. Este proceso mnémico social constituye una forma de memoria colectiva (Halbwachs, 2004), la cual está conformada por los recuerdos y narrativas que se construyen socialmente en torno a lugares que son significativos por su relevancia histórica para ciertos grupos o culturas (Devine-Wright y Lyons, 1997; Low, 1992; Marcus, 1992). Esta construcción social cobra materialidad a través de producciones narrativas (Balasch y Montenegro, 2003; Biglia y Bonet-Martí, 2009), que permiten la exposición y emergencia de los márgenes de posibilidad de acción de un grupo o colectivo.

Se trata de acontecimientos gravitantes, que implican aspectos políticos y formas de participación social (Grau-Solés, ÍñiguezRueda y Subirats, 2011; Íñiguez, 1996) y con ello, modos con que se evalúan dichos cambios, consideraciones relevantes para dimensionar el impacto de un desastre en el sujeto social, lo que no siempre es estudiado al evaluar los cambios asociados ante estas transformaciones.

En resumen, en este trabajo en el que buscamos comprender el impacto del terremoto $8.8^{\circ}$ y tsunami del $27 \mathrm{~F}$, en Dichato, desde la perspectiva de sobrevivientes y habitantes del lugar, recurrimos a herramientas comprensivas trascendiendo la cuantificación o medición del comportamiento, situándonos en el campo de la comprensión de discursos, relaciones y significaciones, donde emergen emociones, memorias y conducta colectiva, siendo la historia relatada, un aspecto fundamental para profundizar respecto a las cualidades o condiciones de posibilidad del bienestar ante graves desastres en el lugar de vida.

\section{Método}

\section{Diseño}

El presente estudio de tipo biográfico (Cornejo, 2006; Pujadas, 1992) se enmarca en una investigación cualitativa (Íñiguez, 1999), cuyo diseño se fundamenta con el objetivo de "entender fenómenos sociales e históricos, para analizar el impacto de intervenciones o cambios sociales a través del análisis de experiencias personales o para reconstruir contextos o épocas de una comunidad" (Berenguera, Fernández de Sanmamed, Pons, Pujol, Rodríguez y Saura, 2014, p. 116). Este tipo de diseño permite el contacto con la 
memoria colectiva, en tanto recuerdos de un pasado que recrean interacciones con terceros, con un lugar significativo, en un entorno sociocultural en particular, definiéndonos en un cierto posicionamiento y trayectoria, a partir de la ocurrencia de un acontecimiento extraordinario que impacta fuertemente en la población.

\section{Participantes}

Dichato es un pueblo de aproximadamente 4000 habitantes (INE, 2012, citado en Contreras y Arriagada, 2016), ubicado a 520 kilómetros al sur de Santiago de Chile y es uno de los principales balnearios de la región del Bío-Bío. Su nombre resuena en Chile al recordar el terremoto de $8.8^{\circ}$ y posterior tsunami, ocurridos en la madrugada del 27 de febrero de 2010, siendo una de las localidades más afectadas por el "tsunami con mayor número de muertes asociada a este tipo de eventos en Sudamérica en los últimos 30 años" (Contreras y Winckler, 2013, p. 8). Dicho acontecimiento significó destrucción, muertes, pérdidas de fuentes laborales, reconstrucción y segregación socioespacial, implicando una serie de cambios según relataron los participantes del estudio que se informa.

En las entrevistas biográficas participaron cinco personas adultas con residencia en Dichato desde su infancia, de edades entre 44 y 77 años, dos mujeres y tres hombres. El muestreo fue intencionado y por bola de nieve (Onwuegbuzie y Leech, 2007), estratificando por sexo y rango etario. Es decir, participantes identificados por su amplia experiencia respecto a lo acontecido, posteriormente vincularon a la presente investigación, a conocidos/as cercanos.

\section{Técnicas}

La investigación efectuada comprendió la utilización de distintas técnicas de producción de información, como observación participante y entrevista biográfica. En el presente artículo sólo se reporta información relativa al proceso de entrevistas.

En la entrevista biográfica (Cornejo, 2006; Cornejo, Mendoza y Rojas, 2008) se solicitó al entrevistado que relatara sus experiencias, vivencias o acontecimientos significativos, recogiendo su propia perspectiva, permitiendo observar el curso de su vida en el lugar, y en particular, conociendo profundamente su mundo tal y como el sujeto lo enuncia (Taylor y Bogdan, 1987). Las entrevistas se llevaron a cabo a través del apoyo de un guión referencial, que contenía ejes temáticos que permitieron la apertura y flexibilidad de respuestas por parte de los participantes (e.g. "Historia del participante en el lugar. Acontecimientos de causa natural o humana de relevancia e implicancias en su historia de vida").

\section{Procedimiento}

Se inició el proceso de observación participante y entrevistas biográficas en el pueblo de Dichato entre septiembre de 2016 y junio de 2017, lo que permitió el acceso a los participantes durante el período indicado. Las técnicas de recopilación de información fueron aplicadas luego de seis años del gran terremoto y tsunami ocurridos en la zona.

El guión de entrevista fue evaluado por profesionales y académicos/as del área de la psicología, y aplicado por el investigador principal de manera abreviada durante el proceso de observación participante. Con el objetivo de probar el guión abreviado de entrevista y contactar a informantes clave, se aplicó lo señalado a tres trabajadores (pescadores y buzo mariscador) del pueblo de Dichato, que tenían la experiencia de haber vivido el desastre del 27F. El propósito principal de lo consignado fue contactar a informantes clave, evaluar la comprensión de preguntas efectuadas y tiempo de duración estimado de su aplicación, para proceder a su aplicación en una segunda oportunidad.

Se efectuó una entrevista biográfica por participante, cuyo foco inicial era explorar la experiencia respecto al $27 \mathrm{~F}$ (reacciones colec- 
tivas, creencias y emociones sociales dominantes, antes y después del acontecimiento señalado).

Las entrevistas fueron realizadas de manera individual en un lapso de tiempo de 6 meses, donde cada una de ellas tuvo una duración de 90 minutos aproximadamente. Cada entrevista fue audiograbada y luego transcripta para su posterior análisis. Se solicitó a los participantes que firmaran una carta de consentimiento informado en la que se explicaba el objetivo de la investigación, así como aspectos de confidencialidad, voluntariedad y anonimato de su participación.

\section{Análisis de información}

Se realizó análisis temático (Braun y Clarke, 2006; Clarke y Braun, 2016), complementado con el método de comparación constante de la teoría fundamentada (Strauss y Corbin, 2002). El análisis temático permite comprender en profundidad el campo de sentido manifiesto y latente que emerge de los/las participantes de la investigación, durante el proceso de recolección de información, entregando con ello aportes para comprender su perspectiva y realidad. Específicamente se identificaron temas, subtemas y nodos, empleándose el software Nvivo 11 para el análisis temático de las entrevistas. Se realizaron múltiples lecturas de las mismas, para luego identificar y seleccionar distintos fragmentos que connotaban alguna acción, situación, objeto o relación de relevancia para los fines de la investigación. Posteriormente, lo seleccionado fue agrupado, organizado y jerarquizado, efectuando análisis e interpretación intracaso y global, siguiendo una lógica de razonamiento inductivo.

\section{Resultados}

Según hemos señalado, para responder al objetivo de investigación, realizamos un análisis temático del corpus textual asociado a los relatos sobre cambios en el lugar de vida. Inicialmente, emergieron recuerdos respecto al 27F, y con posterioridad, relatos asociados a la dictadura militar de Augusto Pinochet (1973-1990), en Chile. En consecuencia, fue en el curso del ejercicio mnémico respecto a la vida de las personas entrevistadas en el lugar, que emergieron de manera espontánea relatos sobre el terrorismo de Estado ejercido durante la Dictadura Militar en Chile asociándose a los hitos que marcaron la vida de las personas con un antes y un después, e impactando en su bienestar.

En ambos acontecimientos, distantes temporalmente entre sí y que presentan diferencias en torno a su origen y su alcance, las emociones sociales dominantes son vinculadas a temor, miedo e incluso terror, producto de relaciones intra e intergrupales que esos mismos acontecimientos facilitaron o acentuaron de manera drástica en su vida cotidiana.

Por lo tanto, los participantes construyeron versiones en que describen y significan prácticas sociopolíticas de autoridades, que contribuyeron a un cambio negativo y traumático en el lugar de vida, un gran daño al sujeto social y a su bienestar, siendo aquello lo que construyó el desastre. A su vez, no se observan políticas de corto, mediano y largo plazo que contribuyan a afrontar lo consignado, más allá de reparaciones materiales, funcionales, estéticas o de infraestructura.

Tras la organización y análisis de la información intracaso, intercaso y global, según temas, subtemas y nodos dominantes, los datos fueron agrupados en tres ejes principales, identificándose a los participantes según sexo (mujer / hombre) y edad. Un eje es relativo al impacto socio-histórico-emocional del desastre, otro eje enseña el daño al sujeto social y por último, el siguiente eje se asocia al bienestar de lugar.

\section{Impacto socio-histórico-emocional (ISHE)}

El primer hallazgo fue que el impacto de un acontecimiento violento no implica necesariamente transformaciones materiales. Los 
hallazgos dan cuenta de un ISHE en el sujeto social de gran magnitud, es decir, un punto de inflexión histórico y emocional compartido, gobernado por el temor, miedo o desconfianza respecto a un otro, según relatos respecto al 27F, como también sobre el golpe militar, vivenciados en el lugar:

Vivencia respecto al 27F:

“(...) de repente todavía me despierto en la noche y siento aún... porque son secuelas que quedan po. (...) hubo gente de los alrededores que vinieron a saquear, a robar, venían con camión, camionetas, vehículos, y robaron. (...) El terremoto nos sirvió para conocernos como persona y ver quiénes somos realmente" (M44).

Vivencia respecto a la dictadura militar: "(Antes del golpe) La gente era muy unida, muy cariñosa, entre todos se ayudaban, pero ahora no. (...), ahora la gente es otra. (...) (Después del 11 de septiembre de 1973) No sabíamos qué iba a pasar, o por último el vecino nos echaba al agua" (H75).

Los discursos se asocian a profundos cambios sociales (relaciones intra e intergrupales), simbólico-imaginarios (generación o reconstrucción de pensamiento, conducta y/o emoción colectiva) e históricos (versiones dominantes en torno a la historia del lugar, sus implicancias y valoración emocional) sobre el lugar de vida.

\section{Daño al sujeto social (DSS)}

De las memorias y acciones en torno a la relación que emerge entre sujetos sociales, luego de graves acontecimientos vivenciados en el lugar específico, se construye el ISHE, y que en este caso significó DSS por el quiebre social que fomentó.

Los relatos describen relaciones intergrupales jerárquicas (basadas en torno al poder, injusticia y egoísmo), que a mediano y largo plazo de ocurrido los acontecimientos, han dañado física y/o socialmente al sujeto social. Principalmente esta relación ha sido con organismos del poder político y económico, que configuran el otro sujeto social situado en posición de poder, que interviene su lugar de vida. En otros términos, a mediano y largo plazo, observan mayor distancia social hacia y desde las autoridades, o bien una aproximación instrumental.

Respecto a prácticas políticas post $27 \mathrm{~F}$ :

"(Sobre la reconstrucción post 27F) Yo creo que si cumplieron el $80 \%$ de lo que estaba en el proyecto, es mucho (...) yo culpo al Serviu (Servicio de Vivienda y Urbanismo), (...) hubo un arreglo con las empresas constructoras, esa es la sinvergüenzura más grande (...) que estas viviendas que estaban construyendo costaban 25 millones de pesos, ¿escuchó bien? El otro compadre que lo hacía de hormigón, cobraba 12 millones y medio... buen negocio po oiga...y aun así, no terminaron bien las cosas" (H77).

Respecto a la dictadura militar:

"Llegaron los marinos, y en la noche disparaban, y tenías que estar encerrado, no podías salir, (...) la gente no se metía en nada, no hablaba nada, temerosa, (...) no se tocaba el tema, y si te pillaban... jcállate, que te pueden estar escuchando!, ¡no hablí de eso, que te pueden estar escuchando!" (M62).

Si bien el sujeto social reconoce el daño e implicancias de lo que ha vivenciado en su lugar de vida, producto de la relación con el otro sujeto social que ahí se ha presentado, naturaliza estas graves experiencias:

"Yo desearía que viniera otra salida de mar. Algunos quedaron más parado todavía, porque así habría más ayuda... Si no hay tsunami no hay ayuda po oiga. (...) porque ahí la autoridad ayuda po (...). Si los desastres hacen para que la autoridad llegue a las partes afectadas" (H75).

\section{Bienestar de lugar (BL)}

Se entiende por BL un proceso de memoria colectiva, relacional y político entre al menos dos sujetos sociales y un lugar. De este proceso surge una valoración emocional sobre experiencias positivas o negativas del sujeto social 
en y con el lugar de vida. Estas versiones sobre el pasado, en el presente y proyección, respecto al lugar de vida, como su aporte al bienestar y salud del sujeto social, construyen el BL. Estas versiones no son homogéneas, mostrando un campo de conflicto al imponerse una de ellas como la dominante. La versión no dominante es la no institucional, que en este caso proviene de los participantes.

Según los relatos, el BL cambió desde el golpe militar de Pinochet. Antes existía una valoración positiva de lo colectivo, de la participación u organización comunitaria, confianza entre sus miembros. Por ejemplo, la conectividad, relaciones y turismo que permitía el ferrocarril, al unir grandes ciudades distantes, y la valoración positiva de las fuentes o industrias de trabajo, en el lugar de vida, nutrían el BL:

"(Antes de la dictadura) la gente trabajaba mucho en las industrias en ese tiempo (...) en el gobierno militar dejó todo eso, echó abajo todo. (...) Aquí lo que más fregó en Dichato fue el ferrocarril (...) Dichato tenía su ganancia con el ferrocarril" (H75).

"Antes (de la dictadura) venía gente de Chillán (en ferrocarril) y acampaban, hacían su fogata en la noche, eso como que se fue perdiendo con el tiempo, porque ahora no se puede acampar, puede estar hasta cierto horario en la playa, hay resguardo policial. (...) (antes) se crearon muchas amistades (...) se conocieron en las fogatas, fue bien bonita esa época. Después con los años el tren se fue eliminando, se eliminó y se eliminó no más" (M44).

Hoy perciben un cambio negativo en el lugar, que se ha ido profundizando a lo largo de los años, donde las dinámicas señaladas y valoradas positivamente fueron eliminadas desde el golpe militar, construyéndose una realidad social extraña, aunque con el tiempo se ha naturalizado.

Los relatos enfatizan un deterioro en el BL, donde el otro sujeto social es percibido como una amenaza, al haber dañado o limitado las posibilidades de mantener el BL, presente y futuro. Lo señalado ha significado falta de fuentes de trabajo, dejar su lugar de vida y familias, proyectar a las futuras generaciones en otras zonas del país:

“(...) aquí no hay trabajo, recién andaba mi hijo menor (...) tiene 31 (años). Perdone, me saqué la cresta para que estudiaran, estudió en la Universidad (...) y ahora no puede encontrar pega po. Está cesante, anda trabajando en la pala, en la construcción, por ahí" (H70).

“(...) nosotros vivimos del turismo en el verano. $\mathrm{Y}$ en el invierno, el que guardó, guardó. Y tú ves aquí, pasa el día y no vendo nada. Entonces en este tiempo para todos es malo" (M62).

Así también, el recuerdo y valoración emotiva respecto del pasado, de cómo era la vida en el lugar, o la valoración positiva de cualidades físicas del entorno, específicamente del mar, entregan aportes positivos al BL:

“(...) (En Dichato) uno sale, conversa, se toma una bebida. (...) Se la toma en la calle, por último sentado, una hora, mirando el mar. Se saca los zapatos, se moja las patas. La vida misma (...) me siento bien aquí" (H70).

“(...) mi vida infantil se puede decir, de niño, fue a la orilla de la costa, (...) íbamos a los roqueríos aquí, a sacar mariscos, otras veces íbamos en bote así, con varillas, a sacar pejerreyes, y otras veces íbamos a calar redes, entonces todo, todo ese vínculo (...) me atrajo al mar, y siempre me ha atraído el mar, en distintas edades y roles (...). Puede ser que se enamore" (H77).

Sin embargo, lo planteado ha sido confrontado por fuertes cambios impuestos en su lugar de vida, significando movilidad, desplazamiento forzado o fuerte tensión, a partir de acontecimientos sociopolíticos y naturales que lo han influenciado:

Sobre la dictadura:

“(...) Pasé el golpe y enseguida me retiré de acá. (...) Yo vivía en un departamento en el norte, el más antiguo de Iquique (ciudad ubicada en el norte de Chile, a 2200 kilómetros de Dichato)" (H75). 
Sobre el 27F:

"Y el mar se metió por ahí para arriba y barrió todo. No quedó nada (...) Impotencia. Oiga, pero si de aquí al portón de ahí andaba un jeep blanco con dos niñitos rubios (...) llamando a la mamá (...) En eso los pilla la ola, y ahí los tiene que haber arrollado el mar. (...) Después del $27 \mathrm{~F}$ la gente se puso más, más agresiva" (H70).

Acontecimientos como la dictadura militar y el $27 \mathrm{~F}$, para participantes de esta investigación, son experiencias extremas con recuerdos vividos, comúnmente guardados en reserva, acontecimientos que los desarticuló socialmente y ha tensionado su BL.

Mientras las dinámicas de cambio ya aludidas se profundizan, comienza a crecer el sector residencial colindante al balneario de Dichato, Pingueral, donde se encuentran amplias casas y se construyen departamentos de lujo. Lo que materialmente divide a ambos sectores, es un portón que prohíbe el ingreso a Pingueral para no residentes, o bien guardias que controlan el acceso al sector y playa. Pingueral es diametralmente distinto en términos socioeconómicos, por su alto poder adquisitivo, siendo la marcada segregación socioespacial una cualidad entre ambos sectores:

"El fundo de Pingueral, que es donde viven los grandes, la gente con plata (...) esa gente, qué se va a fijar en uno po... imagínese. Tienen mansiones, qué se va a fijar en un simple pescador. (...) uno no tiene contacto con esa gente, ellos no están ni ahí. Ellos viven su mundo, en su parte no más. (...) Ese es otro mundo allá, Pingueral es otro mundo" (H75).

"En una oportunidad conversé (con) el que actúa como administrador de ahí (de Pingueral), (...) vino a conversar conmigo acá (a las semanas de ocurrido el tsunami), (...) que él quería arreglar todo esto aquí, (...) y que nosotros le firmáramos y entregáramos todo el terreno, para él poder hacer ese tipo de trabajo y pedir las ayudas al Estado... y yo le pesqué ahí, y le dije..., no..., de mí, no va a obtener firma, yo no lo doy. (...) Todo eso, cuando había pasado el desastre del terremoto, se habían dejado caer. Entonces ipara qué? Para dejarles libre la pasada para ellos y aquí se habrían apoderado y nos habrían echado a todos" (H77).

De acuerdo con los resultados obtenidos en su conjunto, se entienden los desastres no como un hecho aislado y sí desde una mirada sistémica y contextualizada, considerando aspectos sociopolíticos, históricos, culturales y materiales, que construyen y resignifican el sentido y prácticas en torno a un acontecimiento, lo que en su conjunto construye un desastre.

\section{Discusión}

Con el objetivo de comprender el impacto del terremoto $8.8^{\circ}$ y tsunami del 27 de febrero de 2010 en Dichato, desde la perspectiva de sobrevivientes y habitantes del lugar, analizamos relatos sobre las transformaciones en su lugar de vida, toda vez que los lugares aportan hacia la experiencia de bienestar y salud de las personas (Duff, 2009).

Si bien la presente investigación se propuso indagar sobre el impacto del terremoto $\mathrm{y}$ tsunami del 27F, el discurso de los participantes también procuró presentar, a través de recuerdos con carga emocional, otro de los graves acontecimientos ocurrido previamente en el lugar, asociado al terrorismo de Estado en Chile entre 1973 y 1990 y las cualidades o alcances que ahí tuvo.

El 27F y el terrorismo de Estado, siendo acontecimientos de distinta naturaleza, impactaron de manera gravitante, drástica, en la vida de los participantes, mostrando en ambos casos, que lo complejo de lo vivido, luego de la pérdida de vidas y la vulneración de derechos, es el terremoto social, entendido como un cambio drástico en las bases sociales que transforman las relaciones cotidianas, lo que habría promovido emociones sociales dominantes, principalmente miedo y terror, emociones de alerta que luego son naturali- 
zadas o aumenta su tolerancia a las prácticas que promueven aquél terremoto social, en otros términos, existe un cambio en el parámetro con el que evalúan su bienestar, es ahí, donde se construiría el impacto del desastre en este sujeto social. Lo consignado fue recopilado a través de versiones y memorias sobre la vida en el lugar, siendo un aporte a su memoria colectiva.

El estudio del impacto de los acontecimientos, en términos generales, ha aportado identificando el efecto a corto plazo de los desastres (Berroeta, Ramoneda y Opazo, 2015; Cova y Rincón, 2010; Grandón, Acuña, Briese, Chovar, Hernández y Orellanas, 2014). No obstante, el aporte se orientó a comprender el impacto a mediano y largo alcance, en el sujeto social, siendo con ello un aporte innovador. Se identificó un ISHE de gran envergadura, DSS y un detrimento en el BL, al que se arribó al tomar relatos de personas que vivieron dichas experiencias, otorgando un tratamiento cualitativo a la información obtenida.

El ISHE se caracteriza por un punto de inflexión emocional e histórico que significó la imposición de temor, desconfianza y miedo, tras los drásticos acontecimientos. De lo consignado emerge el DSS, que se tradujo en aumento de distancia en las relaciones sociales cotidianas, o bien en la instrumentalización de las mismas, lo que perjudica las posibilidades de comprenderse como comunidad, de promover acción y participación social, o relaciones colaborativas de mediano y largo alcance, ya que el otro, en último término, ha significado ser una amenaza para la estabilidad o conservación del grupo y de la vida en lugar, sean vecinos, actores políticos o de gobierno.

A su vez, la trayectoria de vida en el lugar (Bailey, Devine-Wright y Batel, 2016), del sujeto social, enseña valoraciones positivas sobre el lugar de vida, concentrándose en un pasado colectivo y familiar enriquecedor, que se busca conservar. Por otra parte, tanto en el pasado como en el presente ha existido una valoración positiva del entorno natural.
Así también, se ha mantenido, desde el golpe militar, una valoración negativa respecto a la vida social con pares y autoridades. Es probable que el sentido común impuesto en dictadura, como prácticas sociopolíticas actuales, perpetúen una significación negativa, sobre la participación y organización social, lo que merma el BL.

En consecuencia, es probable que la tensión entre valoraciones antagónicas sobre el lugar de vida, lo material y social, profundice un quiebre en el BL. Dicho quiebre puede vincularse a un trauma colectivo (Kirmayer, Kienzler, Afana y Pedersen, 2010; Somasundaram, 2007), trauma psicosocial (Lykes, 2013; Martín-Baró, 1988) o sufrimiento social (Pedersen, 2002; Pedersen, Kienzler y Gamarra, 2010) de gran envergadura que no se encuentra resuelto, requiriéndose mayores estudios que complementen el presente.

Estudios sobre el bienestar en Chile, enseñan que el bienestar se enmarca en ámbitos de carácter personal o individual (Programa de las Naciones Unidas para el Desarrollo, 2012), lo que este estudio corrobora. Sin embargo, este estudio invita a investigar el BL, que da cuenta del sujeto social, y que en este caso, de mantenerse el tipo de relaciones dañinas entre los sujetos sociales en el lugar, es posible que aumenten la tolerancia o naturalización de lo adverso ante nuevas amenazas.

En su conjunto, los acontecimientos aludidos (terrorismo de Estado y 27F), significaron acciones del Estado y de distintos grupos sociales, que parecen haber cambiado el umbral de tolerancia de los participantes, ante los drásticos y sistemáticos cambios que han impactado en la trayectoria de vida en el lugar, con lo que no sólo cambia el lugar, sino en cómo se evalúan dichos cambios producto del acontecimiento, lo que coincide con lo planteado por Zizek (2016).

Lo que permitiría que los participantes conserven una valoración positiva hacia su lugar de vida, ha sido aislar los hechos significativos que ahí han vivido, asociados principalmente a su familia o bien hacia cualidades 
físicas del lugar, primando una dimensión de vida individual por sobre una colectiva. Por el contrario, la vida colectiva, es decir, el sujeto social, recrea la valoración negativa hacia el lugar de vida, a propósito de las experiencias traumáticas no trabajadas.

En este sentido, la promoción de políticas públicas que favorezcan relaciones comunitarias de participación vinculante y procesos de memoria colectiva, podrían acoger a un desoído sujeto social dañado, comprendiéndose los cambios requeridos en el lugar de vida y las respuestas colectivas, especialmente en zonas de alto riesgo, a modo de facilitar prácticas sociales que logren construir lugares de relevancia, en áreas de protección ante fenómenos naturales, como también procurar transformar relaciones intergrupales, incluidas las relaciones con el Estado.

Por lo tanto, resulta interesante integrar al análisis sobre el impacto de los desastres, el estudio en profundidad del BL, eje analítico traducido en relatos con carga emotiva, que integra memoria colectiva, valoraciones y vínculos positivos y negativos del sujeto social en el lugar vida, todo lo cual se construye socialmente, es decir, entre las comunidades, y abordando la constante tensión entre sujetos sociales, como comunidades y el Estado, u otros grupos en torno al poder.

El estudio que se informa no se encuentra exento de limitaciones. Una de ellas es que se puede complementar con métodos mixtos, considerar distintas poblaciones, permitiendo nutrir la información con una muestra mayor, según objetivos de la investigación.

\section{Conclusión}

Como se dijo, al estudio del impacto de desastres debería integrarse la evaluación y análisis de lo que denominamos ISHE, DSS y BL del sujeto social, lo que implica comprender su impacto a mediano y largo plazo, desde una perspectiva situada.

Lo que este estudio pone en evidencia son las emociones sociales dominantes que emer- gieron en relatos acerca del $27 \mathrm{~F}$, pero que a su vez despiertan los reservados relatos sobre otro hecho traumático ocurrido en años pretéritos, asociado al terrorismo de Estado en Chile, entre 1973 y 1990, cambiando la vida en el lugar.

El ISHE se caracteriza por un punto de inflexión emocional e histórico que significó la imposición de temor, desconfianza y miedo, tras los drásticos acontecimientos. De lo consignado emerge el DSS, que se tradujo en aumento de distancia en las relaciones sociales cotidianas, o bien en su instrumentalización. Por otra parte, el BL ayuda a comprender la valoración emocional sobre experiencias positivas o negativas del sujeto social en y con el lugar de vida. En el caso estudiado, el BL se encuentra altamente mermado.

Es recomendable que futuras investigaciones y políticas públicas, consideren el estudio profundo de los desastres, todo ello a modo de conocer y comprender las respuestas colectivas que pudiesen emerger.

\section{Referencias bibliográficas}

Aravena, A. (2003). El rol de la memoria colectiva y de la memoria individual en la conversión identitaria mapuche. Estudios Atacameños, 26, 89-96. http://dx.doi.org/10.4067/S071810432003002600010

Baeza, M. (2011). Memoria e imaginarios sociales. Imagonautas, 1(1), 76-95.

Balasch, M. y Montenegro, M. (2003). Una propuesta metodológica desde la epistemología de los conocimientos situados: las producciones narrativas. Encuentros en Psicología Social, 1(3), 44-48.

Bailey, E., Devine-Wright, P. y Batel S. (2016). Using a narrative approach to understand place attachments and responses to power line proposals: The importance of life-place. Journal of Environmental Psychology, 48, 200-211.

Batel, S. y Devine-Wright, P. (2015). Towards a better understanding of people's responses to renewable energy technologies: Insights 
from social representations theory. Public Understanding of Science, 24(3), 311-325.

Berenguera, A., Fernández de Sanmamed, M., Pons, M., Pujol, E., Rodríguez, D. y Saura, S. (2014). Escuchar, observar y comprender. Recuperando la narrativa en las Ciencias de la Salud. Aportaciones de la investigación cualitativa. Barcelona: IDIAP J. Gol

Berroeta, H., Ramoneda, A. y Opazo, L. (2015). Sentido de comunidad, participación y apego de lugar en comunidades desplazadas y no desplazadas post desastres: Chaitén y Constitución. Universitas Psychologica, 14(4), 1221-1234. http:// dx.doi.org/10.11144/Javeriana.up14-4. scpa

Biglia, B. y Bonet-Martí, J. (2009). La construcción de narrativas como método de investigación psicosocial. Prácticas de escritura compartida. Forum: Qualitative Social Research, 10(1), 1-25.

Braun V. y Clarke, V. (2006). Using thematic analysis in psychology. Qualitative Research in Psychology, 3(2), 77-101.

Brown, B. y Perkins, D. (1992). Disruptions in place attachment. En I. Altman y S. Low (Eds.), Place attachment (pp.279-304). Nueva York: Plenum Press.

Cárdenas, M., Páez, D., Rimé, B., Bilbao, A. y Asún, D. (2014). Personal emotions, emotional climate, social sharing, beliefs, and values among people affected and unaffected by past political violence. Journal of Peace Psychology, 20(4), 452-464. http:// dx.doi.org/10.1037/pac0000038

Cardona, O. (1993). Evaluación de la amenaza, la vulnerabilidad y el riesgo. En A. Maskrey (Comp.) Los desastres no son naturales. [pp. 45-65] Panamá: Red de Estudios Sociales en Prevención de Desastres en América Latina.

Carnevali, R. (2015). El terrorismo de Estado como violación a los derechos humanos. En especial la intervención de los agentes estatales. Estudios Constitucionales, 13(2), 203-236. http://dx.doi.org/10.4067/S0718-

\section{8}

Clarke, V. y Braun, V. (2016). Thematic analysis. The Journal of Positive Psychology, 12(3), 297-298. http://dx.doi.org/10.1080 /17439760.2016.1262613

Contreras, M. y Winckler, P. (2013). Pérdidas de vidas, viviendas, infraestructura $y$ embarcaciones por el tsunami del 27 de Febrero de 2010 en la costa central de Chile. Obras y Proyectos, 14, 6-19.

Contreras, Y. y Arriagada, C. (2016). Reconstrucción exclusionaria. Lo comunitario y las políticas públicas en ciudades menores e intermedias chilenas afectadas por el terremoto y tsunami del 27F2010. Los casos de Constitución y Dichato. Revista de Geografia Norte Grande, 64, 83-107. http://dx.doi.org/10.4067/ S071834022016000200007

Cornejo, M. (2006). El enfoque biográfico: trayectorias, desarrollos teóricos y perspectivas. Psykhe, 15(1), 95-106.

Cornejo, M., Mendoza, F. y Rojas, R. (2008). La Investigación con Relatos de Vida: Pistas y Opciones del Diseño Metodológico. Psykhe, 17(1), 29-39. http://dx.doi. org/10.4067/S0718-22282008000100004

Cova, F. y Rincón, P. (2010). El terremoto y tsunami del 27-F y sus efectos en la salud mental. Terapia Psicológica, 28(2), 179-185.

Devine-Wright, P. y Lyons, E. (1997). Remembering pasts and representing places: the construction of national identities in Ireland. Journal of Environmental Psychology, 17, 33-45. doi:10.1006/ jevp.1996.0037

Duff, C. (2009). The drifting city: The role of affect and repair in the development of "Enabling Environments". International Journal of Drug Policy, 20(3), 202-208.

Grandón, P., Acuña. S., Briese, C., Chovar, P., Hernández, A. y Orellana, F. (2014). Saqueos y autodefensa. Impacto social en chile post terremoto. Ajayu, 12(2), 187-206.

Grau-Solés, M., Íñiguez-Rueda, L. y Subirats, J. (2011). ¿Cómo gobernar la complejidad? 
Invitación a una gobernanza urbana híbrida y relacional. Athenea Digital, 11(1), 63-84. Halbwachs, M. (2004). Los marcos sociales de la memoria. Chile: Anthropos Editorial. Huber, M. y Arnberger, A. (2015). Opponents, waverers or supporters: the influence of place-attachment dimensions on local residents' acceptance of a planned biosphere reserve in Austria. Journal of Environmental Planning and Management, 59(9), 1610-1628. http://dx.doi.org/10.1080/096 40568.2015.1083415

Íñiguez, L. (1996). Estrategias psicosociales para la gestión de los recursos naturales: De un enfoque individualista a un enfoque social. En L. Íñiguez y E. Pol (Comps.), Cognición, representación y apropiación del espacio [pp.63-81]. España: Universitat de Barcelona.

Íñiguez, L. (1999). Investigación y evaluación cualitativa: bases teóricas y conceptuales. Atención Primaria, 23(8), 496-502.

Jarvis, L. y Lister, M. (2014). State terrorism research and critical terrorism studies: an assessment. Critical Studies on Terrorism, 7(1), 43-61.

Jha, M. (2010). Natural and anthropogenic disasters: An overview. En M.K. Jha (Ed.) Natural and anthropogenic disasters. Dordrecht: Springer.

Kirmayer, L., Kienzler, H., Afana, A. y Pedersen, D. (2010). Trauma and disasters in social and cultural context. En D. Bhugra y C. Morgan (Eds.), Principles of Social Psychiatry [pp. 155-177]. New York: Wiley-Blackwell.

Kõlves, K., Kõlves, K.E. y De Leo, D. (2013). Natural disasters and suicidal behaviours: A systematic literature review. Journal of Affective Disorders, 146, 1-14.

Lewicka, M. (2011). Place attachment: How far have we come in the last 40 years. Journal of Enviromental Psychology, 31, 207-230.

Low, S. (1992). Symbolic ties that bind: place attachment in the plaza. En I. Altman y S. Low (Eds.), Place attachment [pp.165-
184]. Nueva York: Plenum Press.

Low, S. y Altman, I. (1992). Place attachment. En I. Altman y S. Low (Eds.), Place attachment [pp.1-12]. Nueva York: Plenum Press.

Lykes, M.B. (2013). Participatory and action research as a transformative praxis: Responding to humanitarian crises from the margins. American Psychologist, 68(8), 772-783. http://dx.doi.org/10.1037/ a0034360

Marcus, C. (1992). Enviromental memories. En I. Altman y S. Low (Eds.), Place attachment [pp.87-112]. Nueva York: Plenum Press.

Martín-Baró, I. (1988). La violencia política y la guerra como causas del trauma psicosocial en El Salvador. Revista de Psicología de El Salvador, 7(28), 123-141.

Muller, F. y Bermejo, F. (2016). Los temas de la memoria colectiva del golpe de estado de 1976 en Argentina en función de la ideología y las generaciones. Interdisciplinaria, 33(1), 41-63. http://dx.doi.org/10.16888/ interd.2016.33.1.3

Nora, P. (1997). Les lieux de mémoire. Francia: Éditions Gallimard

Norris, F. (2006). Disaster research methods: Past progress and future directions. Journal of Traumatic Stress, 19(2), 173-184. http:// dx.doi.org/10.1002/jts.20109

Norris, F., Friedman, M., Watson, P., Byrne, C., Diaz, E. y Kaniasty, K. (2002). 60.000 disaster victims speak, Part I: An empirical review of the empirical literature, 19812001. Psychiatry, 65, 207-239. http:// dx.doi.org/10.1521/psyc.65.3.207.20173

Oficina de Cambio Climático, Ministerio del Medio Ambiente (2014). Plan Nacional de Adaptación al Cambio Climático. Santiago, Chile: Ministerio del Medio Ambiente.

Onwuegbuzie, A. y Leech, N. (2007). Sampling Designs in Qualitative Research: Making the Sampling Process More Public. The Qualitative Report, 12(2), 238-254. Recuperado de http://nsuworks.nova.edu/ tqr/vol12/iss $2 / 7$ 
Panagioti, M., Gooding, P. y Tarrier, N. (2009). Post-traumatic stress disorder and suicidal behavior: A narrative review. Clinical Psychology Review, 29(6), 471-482. http:// dx.doi.org/10.1016/j.cpr.2009.05.001

Pedersen, D. (2002). Political violence, ethnic conflict, and contemporary wars: Broad implications for health and social well-being. Social Science \& Medicine, 55, 175-190. http://dx.doi.org/10.1016/ S0277-9536(01)00261-1

Pedersen, D., Kienzler, H. y Gamarra, J. (2010). Llaki and Ñakary: Idioms of distress and suffering among the highland quechua in the peruvian Andes. Culture Medicine and Psychiatry, 34, 279-300. http://dx.doi. org/10.1007/s11013-010-9173-z

Piper-Shafir, I., Fernández-Droguett, R. e Íñiguez-Rueda, L. (2013). Psicología social de la memoria: espacios y políticas del recuerdo. Psykhe, 22(2), 19-31.

Programa de las Naciones Unidas para el Desarrollo (2012). Desarrollo Humano en Chile. Bienestar subjetivo: el desafio de repensar el desarrollo. Santiago de Chile.

Pujadas, J.J. (1992). El método biográfico. Las historias de vida en el método biográfico. Madrid: CIS.

Quinn, C. y Halfcare, A. (2014). Place matters: an investigation of farmers' attachment to their land. Human Ecology Review, 20(2), 117-132.

Romero, G. y Maskrey, A. (1993). Cómo entender los desastres naturales. En A. Maskrey (Comp.), Los desastres no son naturales [pp. 6-10]. Panamá: Red de Estudios Sociales en Prevención de Desastres en América Latina.

Sandoval, J. (2013). Una perspectiva situada de la investigación cualitativa en ciencias sociales. Cinta Moebio, 46, 37-46. http://dx.doi.org/10.4067/S0717554X2013000100004

Sapiains, R. y Ugarte, A. (2017a). Contribuciones de la psicología al abordaje de dimensión humana del cambio climático en Chile (Primera parte). Interdisciplinaria, 34(1), 91-106. Recuperado de http://www.scielo.org.ar/pdf/interd/v34n1/ v34n1a06.pdf http://dx.doi.org/10.16888/ interd.2017.34.1.6

Sapiains, R. y Ugarte, A. (2017b). Contribuciones de la psicología al abordaje de dimensión humana del cambio climático en Chile (Segunda parte). Interdisciplinaria, 34(2), 259-274. Recuperado de http://www. ciipme-conicet.gov.ar/ojs/index.php/interdisciplinaria/article/view/332/45 http:// dx.doi.org/10.16888/interd.2017.34.2.2

Sepúlveda-Galeas, M., Sepúlveda, A., Piper, I. y Troncoso, L. (2015). Lugares de memoria y agenciamientos generacionales: lugar, espacio y experiencia. Última Década, 23(42), 93-113. http://dx.doi.org/10.4067/ S0718-22362015000100005

Somasundaram, D. (2007). Collective trauma in northern Sri Lanka: a qualitative psychosocial-ecological study. International Journal of Mental Health Systems, 1(1). http://dx.doi.org/10.1186/1752-4458-1-5

Strauss, A. y Corbin, J. (2002). Bases de la investigación cualitativa. Técnicas y procedimientos para desarrollar la teoría fundamentada. Colombia: Editorial Universidad de Antioquia.

Taylor, S. y Bogdan, R. (1987). Introducción a los métodos cualitativos de investigación. Barcelona: Paidós

Zizek, S. (2016). Acontecimiento. México: Editorial Sexto Piso. 